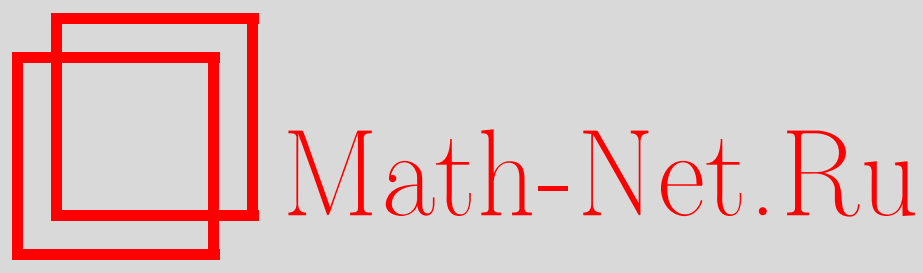

Д. С. Агеев, И. Я. Арефьева, Внезапное нарушение конформной симметрии голографическими сталкивающимися дефектами, ТМФ, 2016, том 189, номер 3, 389-404

DOI: https://doi.org/10.4213/tmf9086

Использование Общероссийского математического портала Math-Net.Ru подразумевает, что вы прочитали и согласны с пользовательским соглашением http://www . mathnet.ru/rus/agreement

Параметры загрузки:

IP: 54.197 .130 .99

26 апреля 2023 г., 10:52:28

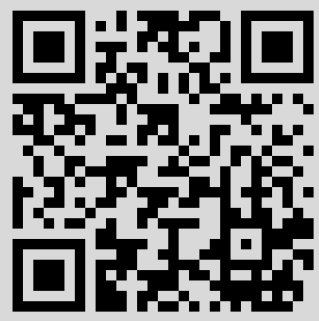




\title{
ФИЗИКА
}

Том 189, № 3

декабрь, 2016

\section{ВНЕЗАПНОЕ НАРУШЕНИЕ КОНФОРМНОЙ СИММЕТРИИ ГОЛОГРАФИЧЕСКИМИ СТАЛКИВАЮЩИМИСЯ ДЕФЕКТАМИ}

\begin{abstract}
Внезапное нарушение конформной симметрии изучается как голографический эффект от ультрарелятивистской частицы, которая движется в пространстве $\mathrm{AdS}_{3}$. Дано качественное описание этого эффекта на основе вычисления двухточечной корреляционной функции и энтропии запутанности соответствующей теории на границе пространства. Показано, что в рамках геодезического приближения безмассовый ультрарелятивистский дефект приводит к зонной структуре коррелятора с нарушенной конформной инвариантностью из-за линзирования геодезических. Голографическая энтропия запутанности также показывает переход к неконформному поведению. Два сталкивающихся ультрарелятивистских дефекта приводят к более сложной зонной структуре корреляторов и энтропии запутанности.
\end{abstract}

Ключевые слова: AdS/CFT-соответствие, голография, конические дефекты, термализация, голографическая энтропия запутанности.

DOI: $10.4213 / \operatorname{tmf} 9086$

\section{1. ВВЕДЕНИЕ}

В настоящей статье мы продолжаем исследовать в рамках AdS/CFT-соответствия двумерную квантовую теорию поля на границе пространства $\mathrm{AdS}_{3}$, деформированного точечными частицами, которые движутся внутри пространства. В предыдущей статье [1] мы изучили деформации теории на границе пространства для случая пространства $\mathrm{AdS}_{3}$, деформированного массивной движущейся частицей. Схожие проблемы рассматривались в более ранних статьях [2]-[5] для различных моделей. Описание мотивации исследования данных моделей см. в работе [1] и цитированной в ней литературе.

Исследование выполнено за счет гранта Российского научного фонда (проект № 14-11-00687).

*Математический институт им. В. А. Стеклова РАН, Москва, Россия. E-mail: ageev@mi.ras.ru, arefeva@mi.ras.ru 
В статьях [1]-[5] для описания конического дефекта применялся теоретико-групповой язык [6]-[8], при этом использовалась определенная процедура разрезания и склейки. Чтобы вычислить двухточечные корреляторы, мы используем геодезическое приближение, предложенное в этом контексте в работе [2]. Ультрарелятивистская точечная частица, начинающая свое движение от границы пространства $\mathrm{AdS}_{3}$ (цилиндра), сжимает внутренность пространства вдоль мировой линии симметрично по отношению к точке начала движения [8]. По мере того как частица проникает внутрь пространства $\mathrm{AdS}_{3}$, геодезические, которые соединяют граничные точки, подвергаются эффекту линзирования. Схожие эффекты имеют место и в случае массивной частицы [1]-[5]; это приводит к появлению зонной структуры в корелляторах теории на границе пространства. Рассматривая столкновение двух ультрарелятивистских частиц, мы получаем еще более сложную структуру геодезических, которая приводит к многозонной структуре двухточечных корреляторов. В частности, в окрестности краев сжимающегося пространства мы получаем фокусировку геодезических, вызванную их нетривиальными намотками вокруг дефекта. Вблизи краев сжимающегося пространства доминируют вклады от наматывающихся геодезических, в то время как вдали от краев доминируют геодезические без намоток. Также существует промежуточная зона, где оба семейства геодезических дают вклад, создавая резонанс. Различные зоны в корреляционных функциях разделены локализованными разрывами, которые распространяются по границе пространства $\mathrm{AdS}_{3}$ с постоянной скоростью.

Изучение голографической энтропии запутанности [9] стало быстро развиваться и обрело широкий спектр приложений в силу относительной простоты вычисления энтропии запутанности в голографии и большого количества модификаций базовых примеров [10]-[15]. В настоящей статье мы вычисляем голографическую энтропию запутанности для двумерных теорий на круге с переменным радиусом. В качестве голографического гравитационного двойника мы рассматриваем те же модели, что и в статье [1], а именно пространство $\mathrm{AdS}_{3}$, деформированное одной или двумя ультрарелятивистскими частицами.

Статья организована следующим образом. В разделе 2 мы даем краткий обзор результатов $\mathrm{AdS}_{3} / \mathrm{CFT}_{2}$-соответствия, которые мы используем для вычислений, а также приводим описание сталкивающихся точечных частиц в пространстве $\mathrm{AdS}_{3}$. В разделе 3 мы вычисляем двухточечную корреляционную функцию, используя геодезическое приближение. В разделе 4 мы находим голографическую энтропию запутанности в присутствии ультрарелятивистских частиц. В заключении мы обсуждаем полученные результаты и направления дальнейших исследований, посвященных столкновениям точечных частиц в голографии.

\section{2. ОСНОВНЫЕ ПОЛОЖЕНИЯ}

Вкратце напомним групповое описание деформации пространства $\mathrm{AdS}_{3}$ ультрарелятивистской частицей [6]-[8].

Точки пространства $\mathrm{AdS}_{3}$ могут быть представлены как элементы группы $S L(2)$ вещественных матриц размера $2 \times 2$ :

$$
\mathbf{x}=x_{3} \mathbf{1}+\sum_{\mu=0,1,2} x^{\mu} \gamma_{\mu}=\operatorname{ch} \chi \boldsymbol{\Omega}(t)+\operatorname{sh} \chi \boldsymbol{\Gamma}(\phi),
$$


где

$$
\mathbf{1}=\left(\begin{array}{ll}
1 & 0 \\
0 & 1
\end{array}\right), \quad \gamma_{0}=\left(\begin{array}{rr}
0 & 1 \\
-1 & 0
\end{array}\right), \quad \gamma_{1}=\left(\begin{array}{ll}
0 & 1 \\
1 & 0
\end{array}\right), \quad \gamma_{2}=\left(\begin{array}{rr}
1 & 0 \\
0 & -1
\end{array}\right)
$$

и

$$
\boldsymbol{\Omega}(t)=\cos t \mathbf{1}+\sin t \gamma_{0}, \quad \boldsymbol{\Gamma}(\phi)=\cos \phi \gamma_{1}+\sin \phi \gamma_{2}
$$

Здесь $(t, \chi, \phi)$ - так называемые “бочечные координаты",

$$
\chi \geqslant 0, \quad \phi \simeq \phi+2 \pi, \quad-\frac{\pi}{2}<t<\frac{\pi}{2} .
$$

Мы также будем использовать координату $r$ диска Пуанкаре, связанную с координатой $\chi$ равенством $r=\operatorname{th}(\chi / 2)$. В координатах диска Пуанкаре метрика пространства $\mathrm{AdS}_{3}$ имеет вид

$$
d s^{2}=-\left(\frac{1+r^{2}}{1-r^{2}}\right)^{2} d t^{2}+\left(\frac{2}{1-r^{2}}\right)^{2}\left(d r^{2}+r^{2} d \phi^{2}\right), \quad r<1 .
$$

Рассмотрим безмассовую частицу со светоподобным вектором импульса, направленным вдоль оси $x$. Ее голономия выражается как

$$
u=1+\operatorname{tg} \epsilon\left(\begin{array}{ll}
0 & 2 \\
0 & 0
\end{array}\right), \quad u^{-1}=1-\operatorname{tg} \epsilon\left(\begin{array}{ll}
0 & 2 \\
0 & 0
\end{array}\right) .
$$

Преобразование изометрии, связанное с голономией, имеет вид

$$
\mathbf{x} \rightarrow \mathbf{x}^{*}=u^{-1} \mathbf{x} u
$$

Мировая линия светоподобной частицы представляет собой множество стационарных точек этой изометрии с $r=\operatorname{tg}(t / 2)$ и $\phi=0$. Чтобы построить область, которую частица вырезает из пространства, будем следовать следующей процедуpe [8]. Рассмотрим пространство $\mathrm{AdS}_{3}$ с точки зрения формализма Арновитта-Дезера-Мизнера (АДМ), так что оно допускает представление в виде эволюционирующего во времени диска Пуанкаре. Далее выделим некоторые кривые $w_{ \pm}$, лежащие в сечениях постоянного времени. Эти кривые отображаются друг на друга под действием введенной изометрии. Наконец, удалим пространство между этими кривыми и отождествим грани удаленного пространства согласно действию изометрии.

Отметим, что частице требуется конечное время, чтобы переместиться из точки границы на диаметрально противоположную сторону. Частица начинает движение в момент времени $t=-\pi / 2$, а заканчивает при $t=\pi / 2$. Мы рассматриваем деформацию пространства только для интервала времени $-\pi / 2<t<\pi / 2$, на этом интервале пространство представляет собой эволюционирующий диск Пуанкаре с вырезанным клином.

Точка $(t, r,-\phi) \in w_{-}$под действием изометрии отображается в $(t, r, \phi) \in w_{+}$. Матрицы, представляющие эти точки, имеют вид

$$
\mathbf{w}_{ \pm}=\frac{1+r^{2}}{1-r^{2}} \boldsymbol{\Omega}(t)+\frac{2 r}{1-r^{2}} \boldsymbol{\Gamma}( \pm \phi)
$$




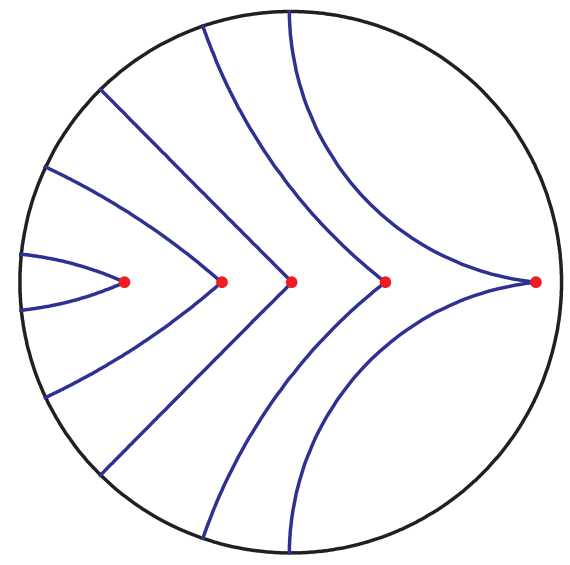

a

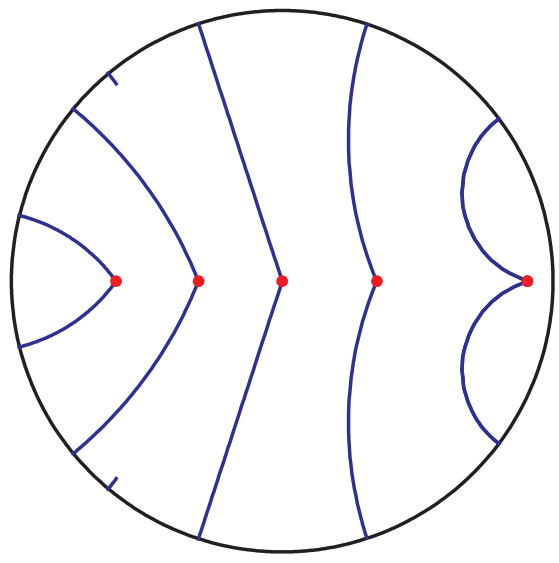

6

Рис. 1. Кривые $w_{ \pm}$для значений параметра $\epsilon=\pi / 4$ (а) и $\epsilon=0.45 \pi$ (б) в различных сечениях постоянного времени. Слева направо на обоих рисунках кривые соответствуют $t=-1.1,-0.4,0,0.3,1.47$.

Записав соотношение $u \mathbf{w}_{+}=\mathbf{w}_{-} u$, находим [8], что грани $w_{+}$и $w_{-}$определяются однозначным образом уравнениями

$$
w_{ \pm}: \quad \frac{2 r}{1+r^{2}} \sin (\epsilon \pm \phi)=\sin t \sin \epsilon
$$

Кривые $w_{+}$и $w_{-}$пересекаются в точке, координаты которой удовлетворяют уравнению $r=\operatorname{tg}(t / 2)$ (задающему светоподобную мировую линию частицы). Эта точка является стационарной точкой изометрии. Пространство получается удалением клина позади частицы между кривыми $w_{ \pm}$и отождествлением граней, заданных уравнениями (9). Получающееся многообразие имеет постоянную кривизну всюду за исключением точек мировой линии частицы.

На рис. 1 изображены кривые $w_{ \pm}$для различных сечений постоянного времени. На рис. 2 показан клин, полученный в результате удаления пространства.

В работе [8] также был исследован случай пространства $\mathrm{AdS}_{3}$, деформированного двумя ультрарелятивистскими частицами, которые начинают свое движение в противоположных точках границы пространства.

Как уже упоминалось, точечные источники деформируют пространство $\mathrm{AdS}_{3}$ локально, так что для каждой частицы и каждой грани клина мы можем применить полученные выше результаты. На рис. За изображен процесс столкновения двух ультрарелятивистских частиц, начинающих движение в точках с угловыми координатами $\phi=0$ и $\phi=\pi$ в момент времени $t=-\pi / 2$, т. е. до момента $t=0$. Пространство между гранями клина слева и справа удалено. Картина после столкновения представлена на рис. 3б. 


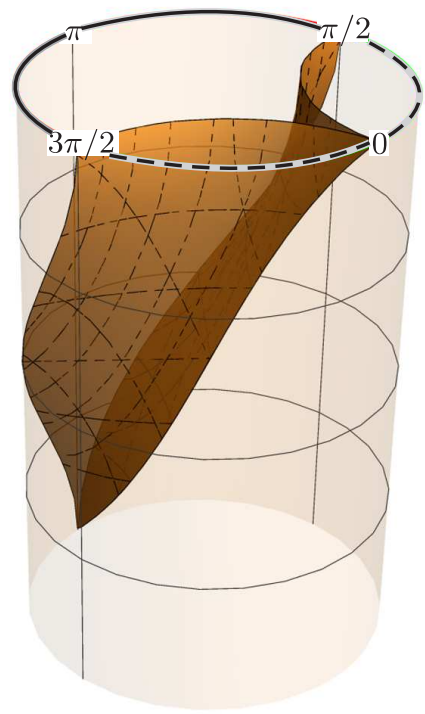

a

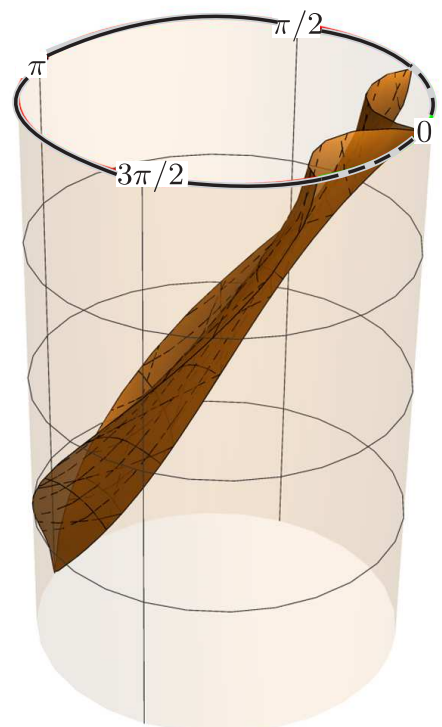

б

Рис. 2. Грани клина, образующегося в результате деформации пространства $\mathrm{AdS}_{3}$ точечной частицей. Жирная линия на границе показывает разрешенную зону для финального момента времени $t=\pi / 2$, штриховая линия соответствует удаленному пространству; значения параметра $\epsilon=\pi / 4$ (а) и $\epsilon=0.45 \pi$ (б).

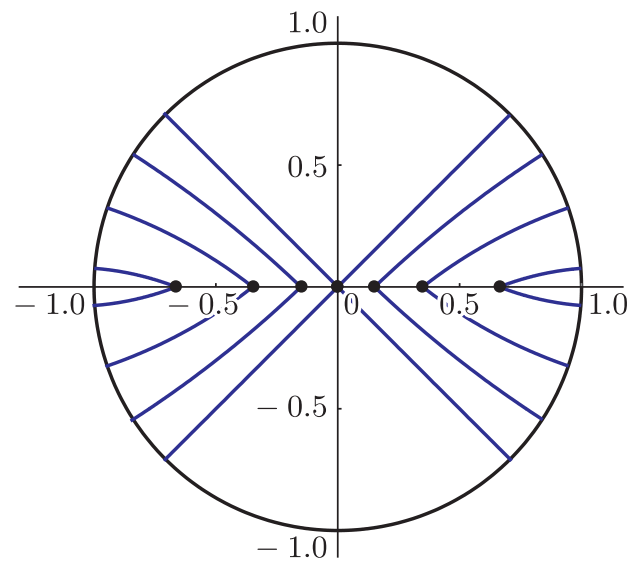

a

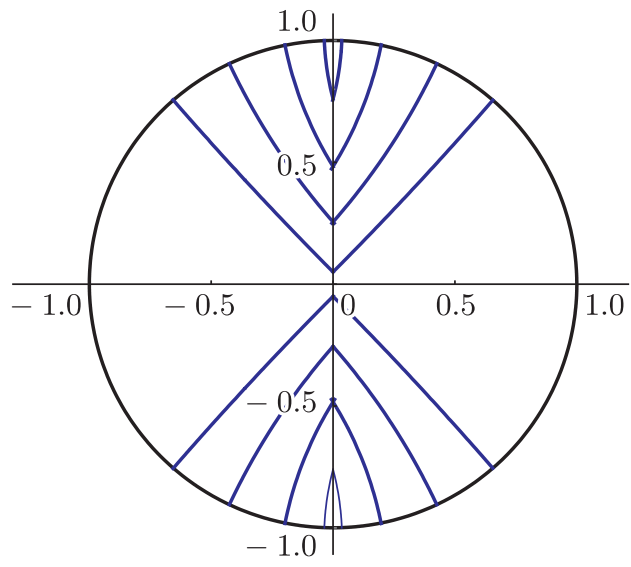

6

Рис. 3. Картина столкновения двух безмассовых точечных частиц до столкновения, в моменты времени $t=-1.17,-0.67,-0.3,0$ (а), и после столкновения, в моменты времени $t=0.1,0.5,0.9,1.3$ (б); значение параметра $\epsilon=\pi / 4$. 


\section{3. КОРРЕЛЯТОРЫ НА ГРАНИЦЕ ПРОСТРАНСТВА $\mathrm{ADS}_{3}$, ДЕФОРМИРОВАННОГО ДВИЖУЩИМИСЯ БЕЗМАССОВЫМИ ЧАСТИЦАМИ}

3.1. Одна безмассовая частица. Рассмотрим двухточечную корреляционную функцию на границе пространства $\mathrm{AdS}_{3}$, деформированного одной безмассовой частицей ${ }^{1)}$. Двухточечную корреляционную функцию на границе пространства $\mathrm{AdS}_{3}$, деформированного безмассовой частицей, можно записать как

$$
\begin{aligned}
& G_{\epsilon, \Delta}\left(\phi_{a}, t_{a}, \phi_{b}, t_{b}\right)=\left(\frac{1}{2\left|\cos \left(t_{a}-t_{b}\right)-\cos \left(\phi_{a}-\phi_{b}\right)\right|}\right)^{\Delta} \Theta_{\mathrm{ncr}}\left(t_{a}, \phi_{a} ; t_{b}, \phi_{b} ; \epsilon\right)+ \\
& +\left(\frac{1}{2\left|\cos \left(t_{a^{\#}}-t_{b}\right)-\cos \left(\phi_{a \#}-\phi_{b}\right)\right|} C_{a^{\#}}^{-1 / 2}\right)^{\Delta} \Theta_{\mathrm{cr}}\left(t_{a}, \phi_{a \#} ; t_{b}, \phi_{b} ; \epsilon\right),
\end{aligned}
$$

где

$$
\begin{aligned}
C_{b^{*}} & =\left(\left(2 \operatorname{tg}^{2} \epsilon+1\right) \sin t_{b}-2 \operatorname{tg} \epsilon \sec \epsilon \sin \left(\epsilon-\phi_{b}\right)\right)^{2}+\cos ^{2} t_{b}, \\
C_{b^{\#}} & =\left(\left(2 \operatorname{tg}^{2} \epsilon+1\right) \sin t_{b}-2 \operatorname{tg} \epsilon \sec \epsilon \sin \left(\epsilon+\phi_{b}\right)\right)^{2}+\cos ^{2} t_{b}
\end{aligned}
$$

являются перенормирующими множителями (см. детали в работе [1]). Функции $\Theta_{\text {nсr }}$ и $\Theta_{\text {сr }}$ определяются следующим образом:

- $\Theta_{\mathrm{ncr}}\left(t_{a}, \phi_{a} ; t_{b}, \phi_{b} ; \epsilon\right)=1$, если геодезические, соединяющие точки $\left(\phi_{a}, t_{a}\right)$ и $\left(\phi_{b}, t_{b}\right)$, не пересекают грани клина ни в какой момент времени;

- $\Theta_{\mathrm{ncr}}\left(t_{a}, \phi_{a} ; t_{b}, \phi_{b} ; \epsilon\right)=0$, если геодезические, соединяющие точки $\left(\phi_{a}, t_{a}\right)$ и $\left(\phi_{b}, t_{b}\right)$, пересекают грани клина в некоторый момент времени;

- $\Theta_{\mathrm{cr}}\left(t_{a}, \phi_{a} ; t_{b}, \phi_{b}\right)=1$, если геодезическая пересекает ближайшую к точке $b$ грань клина в какой-либо момент времени;

- $\Theta_{\mathrm{cr}}\left(t_{a}, \phi_{a} ; t_{b}, \phi_{b}\right)=0$, если геодезическая не пересекает ближайшую к точке $b$ грань клина.

Вычисляя значения функций $\Theta_{\text {cr }}$ и $\Theta_{\text {ncr }}$ численно, мы можем указать, какие именно геодезические дают вклад в коррелятор. Вообще говоря, возможны три варианта конфигураций геодезических, соединяющих две точки на границе:

- существует геодезическая, соединяющая точки $a$ и $b$, которая не пересекает грани клина (мы называем такую геодезическую базовой), и не существует геодезических, наматывающихся вокруг дефекта и соединяющих точки $a$ и $b$ (т. е. не существует геодезической, соединяющей точки $a$ и $b$ и пересекающей клин);

- существует наматывающаяся вокруг дефекта геодезическая и не существует базовой геодезической, соединяющей точки $a$ и $b$;

- существует базовая и наматывающаяся геодезические, соединяющие точки $a$ и $b$.

На рис. 4 и рис. 5 изображены возможные конфигурации геодезических для двух значений параметра $\epsilon$. Если базовая геодезическая не пересекает клин, то она дает вклад в пропагатор, и наоборот, если геодезические, соединяющие образы точек после действия изометрии, не пересекают клин, то они не составляют наматывающуюся геодезическую.

1) Здесь мы подразумеваем деформацию универсального двухточечного коррелятора, заданного уравнением (2.76) в работе [1]. Он связан с коррелятором Вайтмана, запаздывающим, опережающим и причинным корреляторами посредством хорошо известных формул (см. п. 2.3.4 в работе [1]). 


$$
\begin{aligned}
& \varphi_{1}=5.0, t_{1}=0.50 \\
& \varphi_{2}=1.7, t_{2}=0.51
\end{aligned}
$$

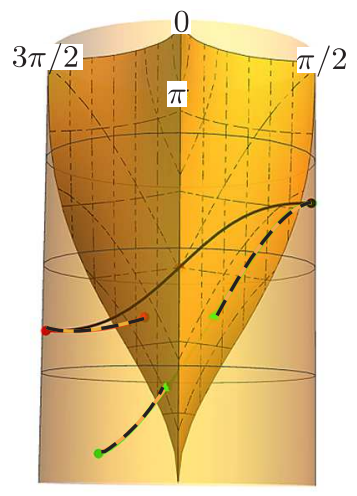

a

$$
\begin{aligned}
& \varphi_{1}=5.0, t_{1}=0.50 \\
& \varphi_{2}=1.0, t_{2}=0.36
\end{aligned}
$$

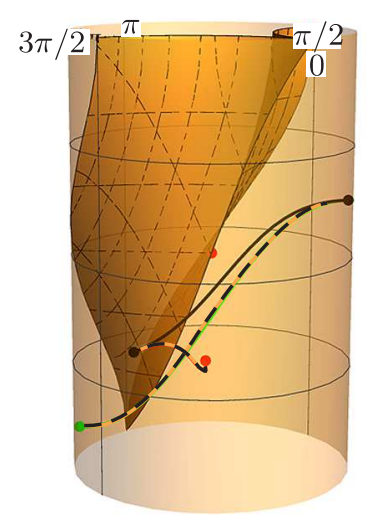

o

$$
\begin{aligned}
& \varphi_{1}=4.4, t_{1}=0.50 \\
& \varphi_{2}=1.6, t_{2}=0.51
\end{aligned}
$$

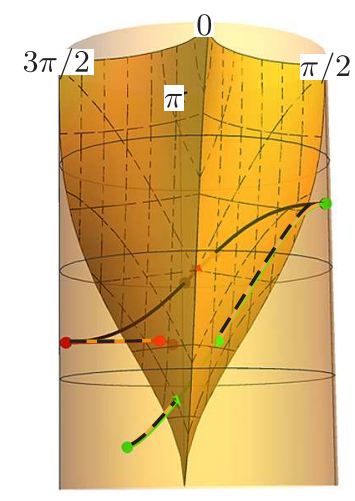

B

Рис. 4. Конфигурации геодезических: жирные кривые являются базовыми геодезическими, штриховые - наматывающимися. Точки на границе с координатами $\left(\phi_{a}, t_{a}\right)$ и $\left(\phi_{b}, t_{b}\right)$ соответствуют концам базовых геодезических. Параметр $\epsilon=\pi / 4$.

$$
\begin{aligned}
& \varphi_{1}=4.8, t_{1}=0.20, \\
& \varphi_{2}=2.0, t_{2}=-0.64
\end{aligned}
$$

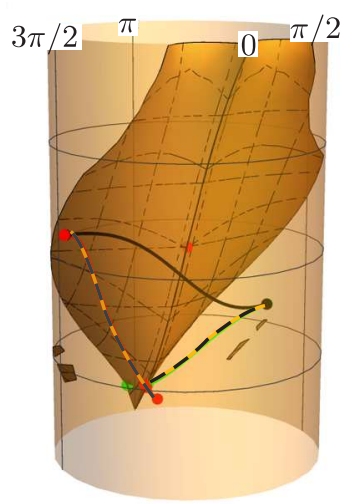

a

$$
\begin{aligned}
& \varphi_{1}=4.8, t_{1}=0.20, \\
& \varphi_{2}=0.86, t_{2}=-0.19
\end{aligned}
$$

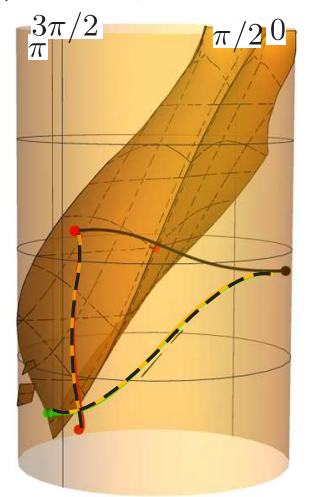

$б$

$$
\begin{aligned}
& \varphi_{1}=4.8, t_{1}=0.20, \\
& \varphi_{2}=1.7, t_{2}=0.023
\end{aligned}
$$

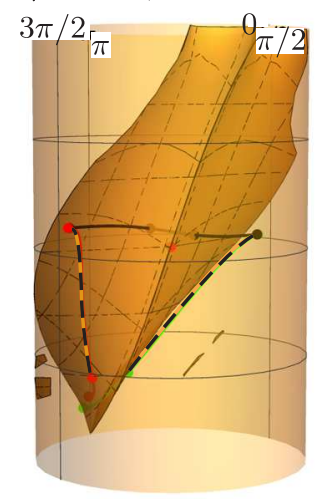

B

Рис. 5. То же, что на рис. 4 , но при $\epsilon=2 \pi / 5$.

На рис. 4а базовая геодезическая (жирная кривая) не пересекает грани клина, наматывающиеся геодезические (штриховые линии) пересекают грани клина и дают вклад в (10). На рис. 4 и базовая, и наматывающаяся геодезические не пересекают грани клина; в этом случае вклад в (10) вносит только геодезическая без намотки. На рис. 4в базовая геодезическая и геодезические, соединяющие соответствующие образы точек на границе после действия изометрии, пересекают грани клина, таким образом, вклад в (10) вносит только наматывающаяся геодезическая. Такая же картина имеет место для геодезических, представленных на рис. 5. 

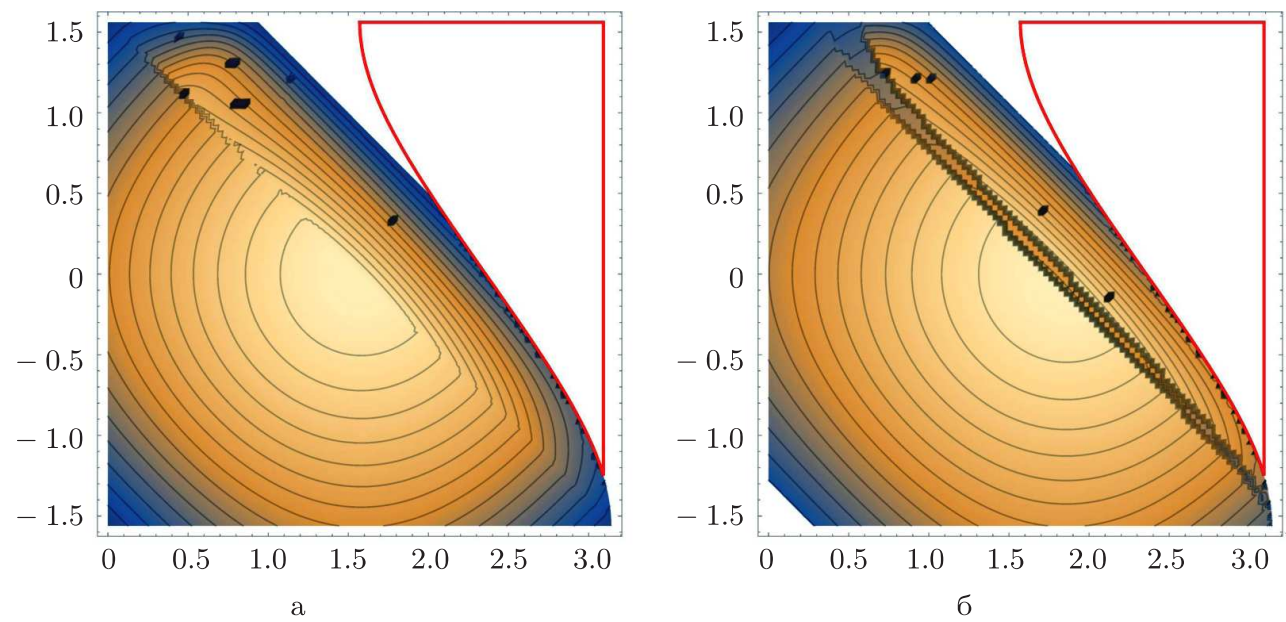

Рис. 6. Графики функции (11). Угловая координата ф отложена по горизонтальной оси, а временная координата $t$ - по вертикальной. Параметр $\epsilon=\pi / 4$. Жирная замкнутая кривая соответствует границе жизненного пространства. Графики нарисованы для $\phi_{1}=3 \pi / 2(\mathrm{a})$ и $\phi_{1}=5(б)$, время $t_{1}=0$, конформная размерность $\Delta=1$. Жирные зигзагообразные линии, пролегающие рядом с диагональю на каждом из рисунков, соответствуют разрывам в корреляторе, которые возникают из-за присутствия различных вкладов.
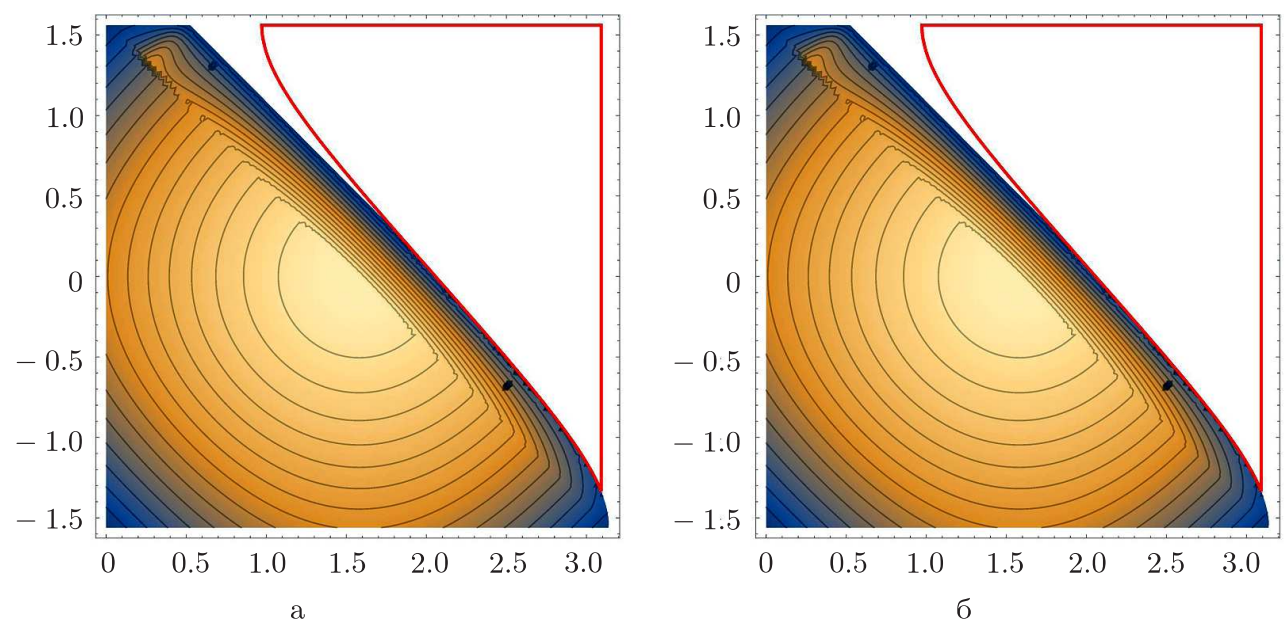

Рис. 7. То же, что на рис. 6 , но для $\epsilon=1$. 
Рассмотрим влияние движущейся частицы на двухточечную корреляционную функцию $G_{\epsilon, \Delta}\left(\phi_{1}, t_{1}, \phi_{2}, t_{2}\right)$. Выберем одну из точек, например $\left(\phi_{1}, t_{1}\right)$, и зафиксируем ее. Предположим, что углы $\phi_{1}$ и $\phi_{2}$ отвечают точкам, расположенным на противоположных относительно мировых линий частиц сторонах границы деформированного пространства $\mathrm{AdS}_{3}$, т. е. $\phi_{1} \in(\pi, 2 \pi)$ и $\phi_{2} \in(0, \pi)$, при этом времена $t_{1}, t_{2} \in(-\pi / 2, \pi / 2)$. Изучим качественное поведение функции

$$
\mathcal{G}_{\epsilon, \Delta, \phi_{1}, t_{1}}(\phi, t)=G_{\epsilon, \Delta}^{-1}\left(\phi_{1}, t_{1}, \phi, t\right) .
$$

На рис. 6 и рис. 7 изображены двумерные графики функции $\mathcal{G}_{\epsilon, \Delta, \phi_{1}, t_{1}}(\phi, t)$ для различных значений $\epsilon, t_{1}$ и $\phi_{1}$. Рядом с краем жизненного пространства видны нетривиальные изменения в корреляторе. Мы называем далее эту зону пульсом. Пульс распространяется от краев дефекта и изменяет свои размеры со временем. На рис. 6а мы можем видеть, что образуются разрывы в момент $t=-\pi / 2$ рядом с точкой на границе, из которой частица начинает свое движение, далее эти разрывы распространяются с практически постоянной скоростью. Слева от разрыва коррелятор не подвержен изменению и его конформная симметрия не нарушена. На рис. $6 б$ мы видим, каким образом появляются эти разрывы и возникает резонанс из-за смешивания различных вкладов от геодезических.

3.2. Две сталкивающиеся безмассовые частицы. Рассмотрим две сталкивающиеся точечные частицы в пространстве $\mathrm{AdS}_{3}$. Это случай включает в себя больше возможностей для конфигураций геодезических, наматывающихся вокруг центра дефекта, который создается парой безмассовых частиц, и картина корреляторов в данном случае усложняется.

Мы имеем два общих случая. Сначала возьмем две точки на противоположных частях цилиндра симметрично по отношению к линии столкновения, т. е. рассмотрим коррелятор $G\left(\phi_{1}, t_{1}, \phi_{2}, t_{2}\right)$ при $\pi<\phi_{1}<2 \pi$ и $0<\phi_{2}<\pi$. Время лежит в интервале $-\pi / 2<t_{1}, t_{2}<\pi / 2$. В этом случае вклад вносят два типа геодезических: базовая геодезическая и геодезическая, проходящая через грани каждого дефекта один раз (см. рис. 8).

Второй случай возникает, когда мы берем две точки на одной границе пространства, т. е. $\pi<\phi_{1}<2 \pi$ и $\pi<\phi_{2}<2 \pi$. В этом случае двухточечный коррелятор получает два вклада, один от базовой геодезической, второй от геодезической, проходящей через грани разных клинов. Сначала геодезическая проходит через нижнюю грань левого клина, а затем через верхнюю грань правого клина. Схематическая иллюстрация этого случая представлена на рис. 9.

В настоящей статье мы рассматриваем только первый случай. Он соответствует эффектам, связанным с большими расстояниями в дуальной теории. Универсальный коррелятор в случае, когда частицы находятся на противоположных сторонах пространства $\mathrm{AdS}_{3}$, имеет вид

$$
\begin{aligned}
& G\left(\phi_{a}, t_{a}, \phi_{b}, t_{b}\right)_{\epsilon_{1}, \epsilon_{2}, \Delta}=\left(\frac{1}{2\left|\cos \left(t_{a}-t_{b}\right)-\cos \left(\phi_{a}-\phi_{b}\right)\right|}\right)^{\Delta} \Theta_{\mathrm{ncr}}^{\text {all }}\left(a, b ; \epsilon_{1}\right) \Theta_{\mathrm{ncr}}^{\text {all }}\left(a ; b ; \epsilon_{2}\right)+ \\
& +\left(\frac{1}{2\left|\cos \left(t_{a^{\#}}-t_{b}\right)-\cos \left(\phi_{a^{\#}}-\phi_{b}\right)\right|} C_{a^{\# 1}}^{-1 / 2}\right)^{\Delta} \Theta_{\mathrm{cr}}^{\ell \ell}\left(a^{\#}{ }_{1}, b ; \epsilon_{1}\right) \Theta_{\mathrm{ncr}}^{\ell \mathrm{u}}\left(a, b^{* 1} ; \epsilon_{2}\right)+ \\
& +\left(\frac{1}{2\left|\cos \left(t_{a}-t_{b_{2}}\right)-\cos \left(\phi_{a}-\phi_{b_{2}}\right)\right|} C_{a^{\#}}^{-1 / 2}\right)^{\Delta} \Theta_{\mathrm{cr}}^{\mathrm{r} \ell}\left(a^{\# 2}, b ; \epsilon_{2}\right) \Theta_{\mathrm{ncr}}^{\mathrm{ru}}\left(a ; b^{\#_{2}} ; \epsilon_{1}\right)
\end{aligned}
$$




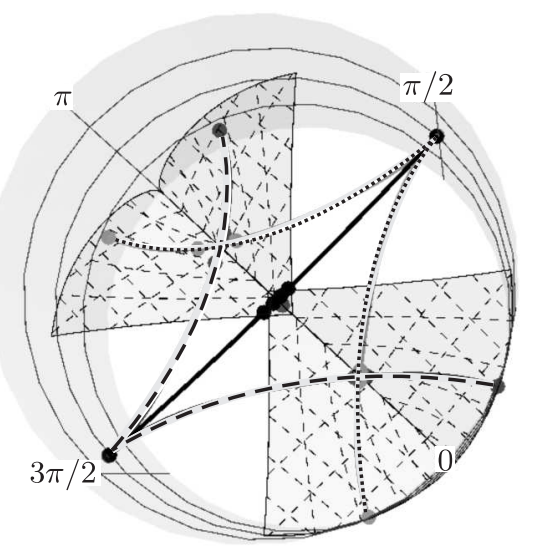

Рис. 8. Различные геодезические конфигурации для двух выделенных точек (концы жирных линий) на границе. Жирная кривая является базовой геодезической, штриховые геодезические проходят через левую и правую грань клина, штрихпунктирные геодезические - их образы под действием изометрии (здесь $\epsilon=\pi / 4$ для обоих дефектов).

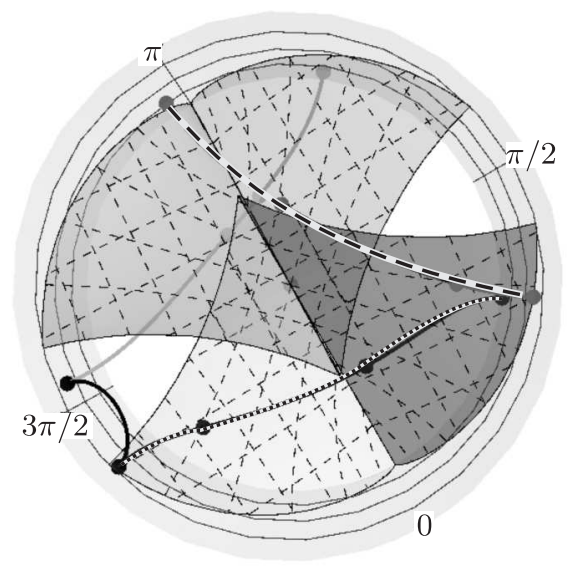

Рис. 9. Один из возможных дополнительных вкладов в коррелятор, когда точки на границе (концы жирной базовой геодезической) лежат на разных сторонах границы. Геодезические проходят через клины. Штриховая линия отвечает промежуточному случаю: она является результатом применения изометрии, индуцированной левым клином, к геодезической, изображенной тонкой линией. Штрихпунктирная геодезическая является результатом применения изометрии, индуцированной правым клином, к штриховой геодезической. Значение параметра $\epsilon=\pi / 4$ для обеих частиц. 

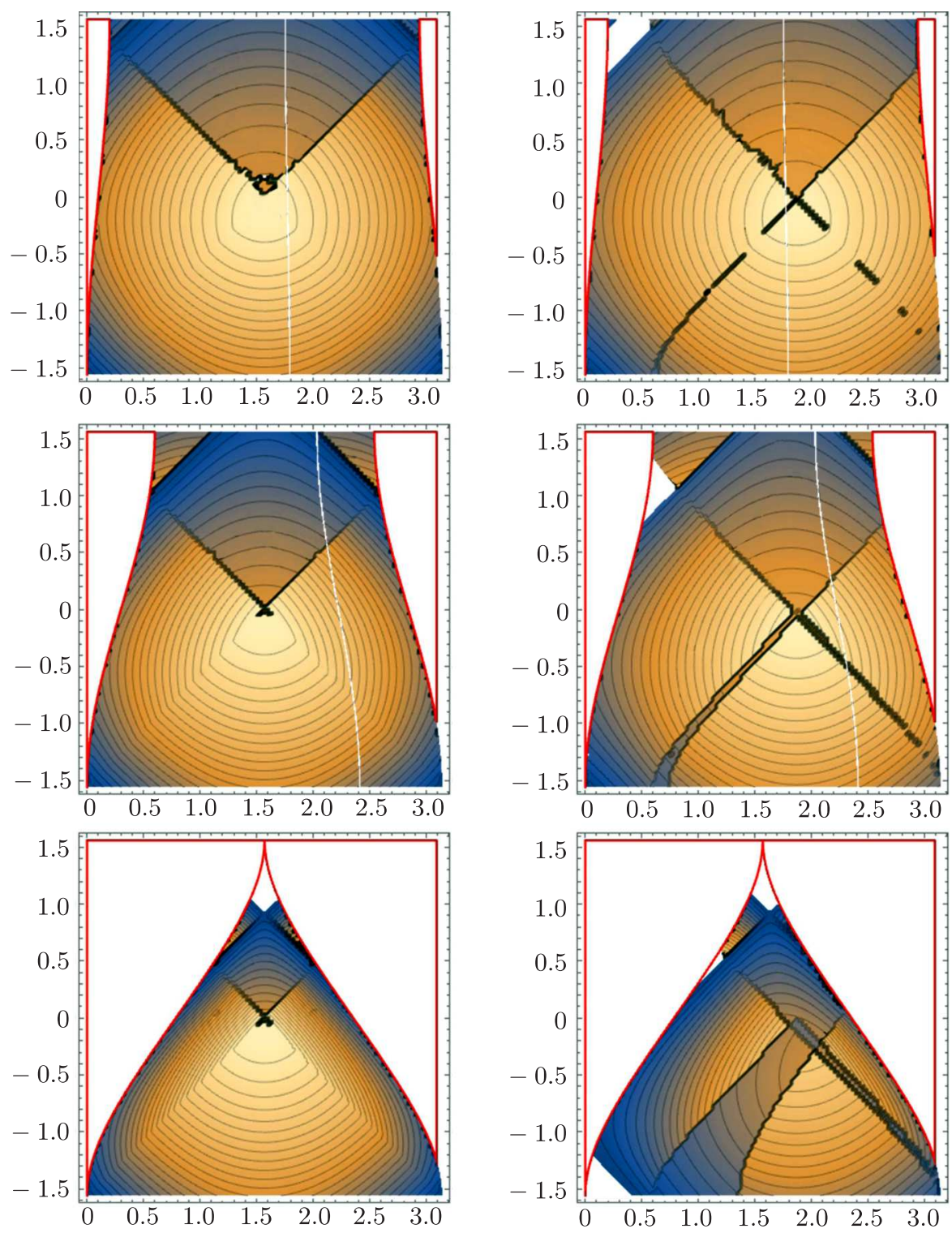

Рис. 10. Графики обратных корреляторов в случае столкновения двух безмассовых частиц для $\phi_{1}=3 \pi / 2$ (левый столбец) и $\phi_{1}=5$ (правый столбец) при $t_{1}=0$. Конформная размерность $\Delta=1$ для каждого графика. По столбцам $\epsilon=0.1,0.3,0.78$ сверху вниз. Жирные кривые соответствуют границам запрещенных зон (где пространство удалено), зигзагообразные кривые соответствуют разрывам, разделяющим пульсы. 

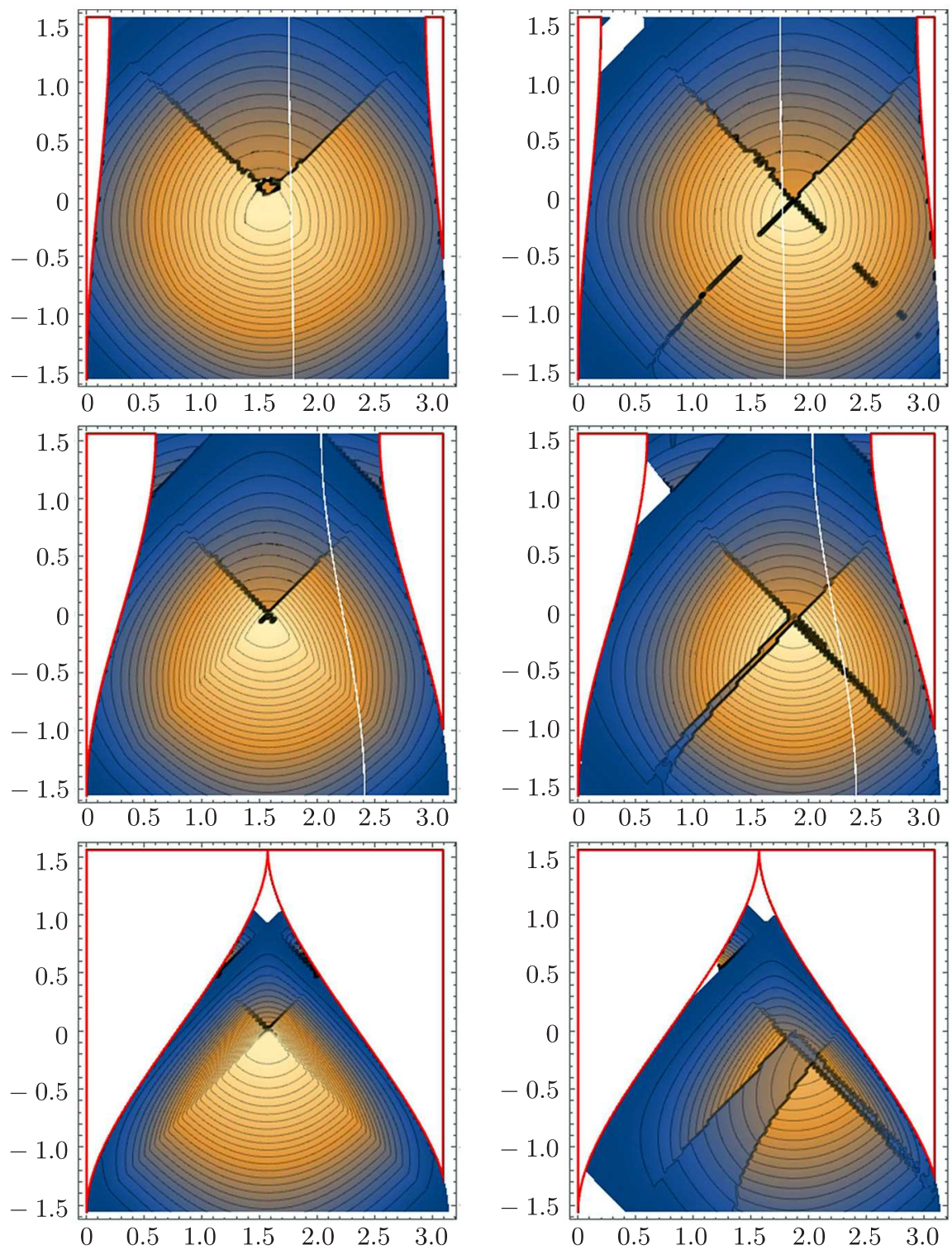

Рис. 11. То же, что на рис. 10, но при $\Delta=2$. 
Первое слагаемое в правой части равенства соответствует вкладу от базовой геодезической, второе - вкладу от геодезической, проходящей через левый клин, третье описывает вклад от геодезических, проходящих через правый клин. Здесь мы используем функции $\Theta$, определенные следующим образом:

- $\Theta_{\mathrm{ncr}}^{\text {all }}(a, b ; \epsilon)=1$, если геодезическая, соединяющая точки $a$ и $b$, не пересекает любой клин, иначе $\Theta_{\mathrm{ncr}}^{\text {all }}(a, b ; \epsilon)=0$;

- $\Theta_{\mathrm{cr}}^{\ell \ell}(a, b ; \epsilon)=1$, если геодезическая пересекает левую нижнюю грань клина, иначе $\Theta_{\mathrm{cr}}^{\ell \ell}(a, b ; \epsilon)=0$;

- $\Theta_{\mathrm{cr}}^{\mathrm{r} \ell}(a, b ; \epsilon)=1$, если геодезическая пересекает правую нижнюю грань клина, иначе $\Theta_{\mathrm{cr}}^{\mathrm{r} \ell}(a, b ; \epsilon)=0$;

- $\Theta_{\mathrm{ncr}}^{\ell \mathrm{u}}(a, b ; \epsilon)=1$, если геодезическая не пересекает левую верхнюю грань клина, иначе $\Theta_{\mathrm{ncr}}^{\ell \mathrm{u}}(a, b ; \epsilon)=0$;

- $\Theta_{\mathrm{ncr}}^{\mathrm{ru}}(a, b ; \epsilon)=1$, если геодезическая не пересекает правую верхнюю грань клина, иначе $\Theta_{\text {ncr }}^{\text {ru }}(a, b ; \epsilon)=0$.

На рис. 10 и рис. 11 мы представляем двумерные графики обратных корреляторов на границе для случая двух безмассовых частиц при $\phi_{1}=3 \pi / 2$ и $\phi_{1}=5$. На всех этих графиках мы видим две зоны пульса, распространяющиеся от каждой границы. Наблюдается асимметрия графиков из правых столбцов на рис. 10 и рис. 11, которая связана с асимметрией позиции точки $\phi_{1}$ по отношению к линии столкновения. В некоторый момент времени эти два пульса сталкиваются, формируя новую структуру. Отметим, что асимметрия сохраняется в течение всего процесса столкновения.

\section{4. ВЫЧИСЛЕНИЕ ГОЛОГРАФИЧЕСКОЙ ЭНТРОПИИ ЗАПУТАННОСТИ}

4.1. Один безмассовый дефект. В этом пункте мы вычисляем энтропию запутанности для пространственноподобных интервалов и различных одновременных сечений в нашей фоновой метрике. Мы фиксируем одновременные точки на противоположных по отношению к мировой линии частицы частях границы пространства $\mathrm{AdS}_{3}$, а именно полагаем $t_{1}=t_{2}=t_{0}$. Чтобы вычислить энтропию запутанности, мы изменяем $t_{0}$ от $-\pi / 2$ до $\pi / 2$. Для статического пространства голографическая энтропия запутанности [10] равна ${ }^{2)}$ минимальной перенормированной геодезической длине $L_{\mathrm{ren}}\left(\phi_{1}, t_{0}, \phi_{2}, t_{0}\right)$ между двумя точками $\left(\phi_{1}, t_{0}\right)$ и $\left(\phi_{2}, t_{0}\right)$ на границе пространства,

$$
S\left(\phi_{1}, \phi_{2}, t_{0}\right)=\min \mathcal{L}_{\text {ren }}\left(\phi_{1}, t_{0}, \phi_{2}, t_{0}\right)
$$

В силу того что мы можем отделить пространственное направление от временного с помощью формализма АДМ, в этой фоновой метрике применима формула (13). На рис. 12 показано, каким образом можно измерить пульсы, описанные в предыдущем разделе, с помощью голографической энтропии запутанности. На этом рисунке показана зависимость голографической энтропии запутанности от $t_{0}$ и $\phi_{2}$ для фиксированного $\phi_{1}$ при различных $\epsilon$. Мы видим что подобно случаю двухточечных функций существует расширяющийся пульс, распространяющийся от начальной точки движения частицы на границе. Видно, что в течение некоторого времени

2) Мы опускаем такие множители в выражении для голографической энтропии запутанности, как гравитационная постоянная и т. п. 


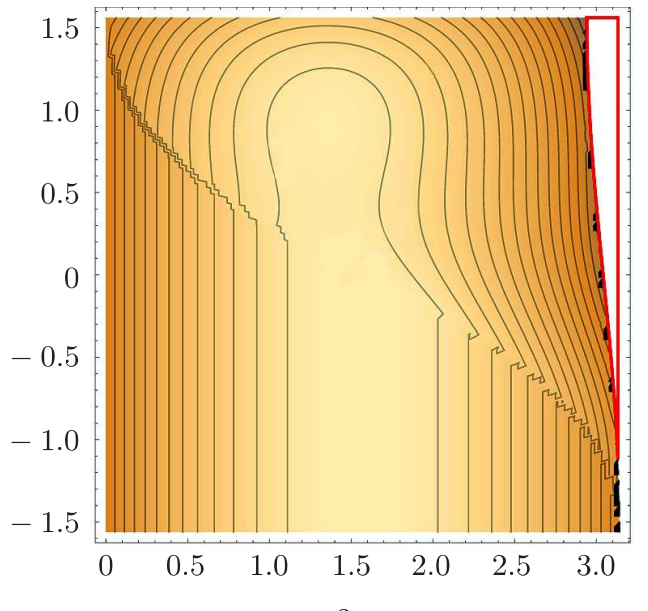

a

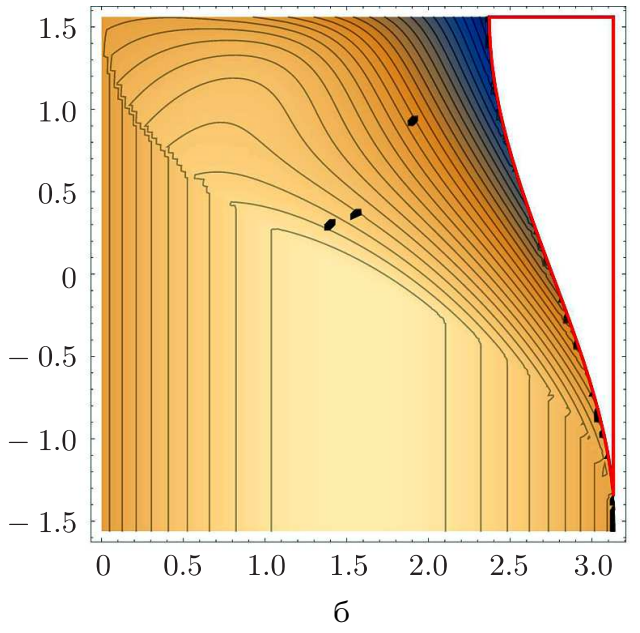

6

Рис. 12. Зависимость голографической энтропии запутанности (13) от $t_{0}$ и $\phi_{2} ;$ здесь $\phi_{1}=3 \pi / 2$, параметр $\epsilon=0.78$ (а) и $\epsilon=0.38$ (б).
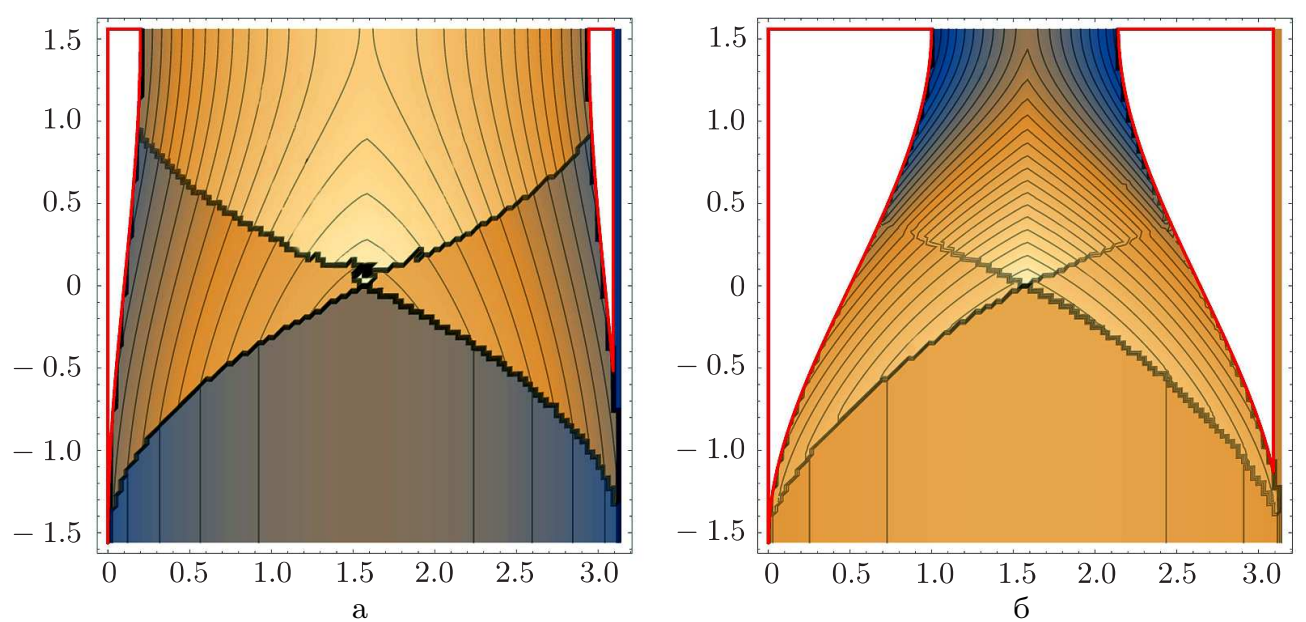

Рис. 13. То же, что на рис. 12, но при $\epsilon=0.1$ (а) и $\epsilon=0.5$ (б).

голографическая энтропия запутанности остается постоянной, а затем происходит резкий переход к неравновесному режиму. Для бо́льших интервалов данный переход происходит быстрее.

4.2. Две сталкивающиеся частицы. В этом пункте мы рассматриваем эволюцию голографической энтропии запутанности в пространстве $\mathrm{AdS}_{3}$, деформированном двумя сталкивающимися безмассовыми частицами.

Вновь мы имеем два различных случая: когда рассматривается эволюция голографической энтропии запутанности для пространственноподобного интервала, концы которого лежат по разные (относительно линии столкновения) стороны границы, и когда эти точки находятся на одной стороне границы. Возникающие при 
этом конфигурации геодезических те же, что и в случае двухточечного коррелятора, однако теперь вместо суммы по всем геодезическим выбирается конфигурация, обладающая минимальной перенормированной длиной.

На рис. 13 показано, каким образом голографическая энтропия запутанности отражает процесс столкновения двух частиц: изображена зависимость энтропии от $t_{0}$ и $\phi_{2}$ для фиксированного $\phi_{1}$ и различных значений параметра $\epsilon$. В данном случае жирные контуры соответствуют сжимающейся границе жизненного пространства. На рис. 13а мы видим широкие зоны, разделенные разрывами (зигзагообразные кривые), отходящие от каждой границы. Размеры этих зон быстро возрастают, и в некоторый момент времени режим еще раз меняется во всем жизненном пространстве. Кривые на рис. 13а рассчитаны при $\epsilon=0.1$. На рис. $13 б$ энергия больше, $\epsilon=0.5$, видно, что размеры и скорость роста зон относительно слабо зависят от $\epsilon$.

\section{5. ЗАКЛЮЧЕНИЕ}

В предложенной статье мы рассмотрели модели квантовой теории поля, дуальной пространству $\mathrm{AdS}_{3}$ с одной ультрарелятивистской точечной частицей, а также процессы столкновения таких частиц в пространстве $\mathrm{AdS}_{3}$. В обоих случаях дуальные модели живут в пространствах с переменными размерами. Для таких моделей мы изучили двухточечные корреляционные функции в рамках геодезического приближения и голографическую энтропию запутанности. Наши численные расчеты показывают, что, используя эти модели, можно уловить некоторые особенности квантовых систем, подвергнутых внезапному эффекту квенча (гашения), и квантовых систем с переменным объемом.

Мы показали, что в рамках геодезического приближения ультрарелятивистские безмассовые дефекты порождают зонную структуру вследствие эффекта линзирования геодезических. Нестационарность пространства обеспечивает существование волн возбуждений, двигающихся вдоль границ от точек квенча (точек, откуда начинают движение частицы). Распространяющаяся зона пульса локализована вблизи концов клина на границе. Также существуют промежуточные зоны, разделенные локализованными разрывами, которые распространяются с постоянной скоростью вдоль границы жизненного пространства. Голографическая энтропия запутанности также имеет нетривиальную зонную структуру. Два сталкивающихся безмассовых дефекта порождают более сложную зонную структуру корреляторов и энтропии запутанности.

Интересно было бы понять, являются ли эти разрывы в коррляторе и энтропии артефактами примененного нами классического приближения или они присутствуют в полном решении, полученном с использованием скалярных полевых уравнений. Мы планируем исследовать этот вопрос отдельно в нашей будущей работе.

Результаты данной статьи представляют интерес для исследования голографическим методом как физики маломерных систем [16], так и физики многомерных сильно коррелированных систем [17], [18], от физики кварк-глюонной плазмы, [19], [20] до фотосинтеза [21].

Благодарности. Авторы благодарны Андрею Багрову, Михаилу Храмцову, Марии Тихановской и Игорю Воловичу за полезные обсуждения в ходе работы над статьей. 


\section{Список литературы}

[1] И. Я. Арефьева, М. А. Храмцов, М. Д. Тихановская, ТМФ, 189:2 (2016), 296-311, arXiv: 1512.03363.

[2] V. Balasubramanian, S.F. Ross, Phys. Rev. D, 61:4 (2000), 044007, 12 pp., arXiv: hep-th/9906226.

[3] И. Я. Арефьева, А. А. Багров, ТМФ, 182:1 (2015), 3-27.

[4] V. Balasubramanian, B. D. Chowdhury, B. Czech, J. de Boer, JHEP, 01 (2015), 048, 22 pp., arXiv: 1406.5859 .

[5] I. Arefeva, A. Bagrov, P. Saterskog, K. Schalm, Phys. Rev. D, 94:4, 044059, 18 pp., arXiv: 1508.04440 .

[6] S. Deser, R. Jackiw, G. 't Hooft, Ann. Phys., 152:1 (1984), 220-235.

[7] G. 't Hooft, Class. Quantum Grav., 13:5 (1996), 1023-1039, arXiv: gr-qc/9601014.

[8] H.-J. Matschull, Class. Quantum Grav., 16:3 (1999), 1069-1095, arXiv: gr-qc/9809087.

[9] S. Ryu, T. Takayanagi, Phys. Rev. Lett., 96:18 (2006), 181602, 4 pp., arXiv: hep-th/0603001.

[10] T. Nishioka, S. Ryu, T. Takayanagi, J. Phys. A: Math. Theor., 42:50 (2009), 504008, 35 pp., arXiv: 0905.0932.

[11] C. T. Asplund, A. Bernamonti, F. Galli, T. Hartman, JHEP, 02 (2015), 171, 23 pp., arXiv: 1410.1392 .

[12] M. Nozaki, T. Numasawa, T. Takayanagi, JHEP, 05 (2013), 080, 38 pp., arXiv: 1302.5703.

[13] D. Marolf, H. Maxfield, A. Peach, S. F. Ross, Class. Quantum Grav., 32:21, 215006, 29 pp., arXiv: 1506.04128.

[14] H. Casini, H. Liu, M. Mezei, JHEP, 07 (2016), 077, 63 pp., arXiv: 1509.05044.

[15] T. Hartman, N. Afkhami-Jeddi, Speed limits for entanglement, arXiv: 1512.02695.

[16] I. Ya. Aref'eva, M. A. Khramtsov, JHEP, 04 (2016), 121, 22 pp., arXiv: 1601.02008.

[17] I. Aref'eva, AIP Conf. Proc., 1701 (2016), 090001.

[18] И. Я. Арефьева, ТМФ, 184:3 (2015), 398-417.

[19] I. Aref'eva, EPJ Web Conf., 125 (2016), 01007, 12 pp.

[20] D. Ageev, EPJ Web Conf., 125 (2016), 04007, 6 pp.

[21] I. Ya. Aref'eva, I. Volovich, Holographic photosynthesis, arXiv: 1603.09107.

Поступила в редакцию 27.10.2015 